\title{
Research on Model of Feasible Timing of Traffic Light for Intersection Control
}

\author{
Jiangqun Wang ${ }^{1}$, Ning Cao ${ }^{1}$, Guozhong $\mathrm{Yao}^{2}$ \\ ${ }^{1}$ Beijing Institute of Technology, Beijing, China \\ ${ }^{2}$ Kunming University of Science and Technology, Kunming, China
}

Keywords: Vehicles queued feasible traffic light cycle.

\begin{abstract}
Traffic lights play a significant role in regulating traffic flow. Due to the use of stationary phase and fixed cycle in traditional systems, it is not feasible to adjust the phase time to meet realtime traffic demand, resulting in delays, inefficiencies and other losses for traffic participants. This paper presents a feasible mathematical model of traffic signal control based on real-time vehicle detection. Two sensors are used to obtain important parameters of the vehicle queue, such as queue length, the number of vehicles queued, the number of vehicles passing the intersection and reaching the intersection during the green light time, the average speed of the vehicle and so on. These parameters are used to calculate actual demand time and feasible traffic light cycle. The feasible traffic light cycle can reduce the delay time for the car to pass the intersection and shorten the queue length. This model depends on the real-time characteristics of the queue, which is much closer to actual situation. Other traffic participants are considered as the boundary constrains of the model.
\end{abstract}

\section{Introduction}

The problems caused by traffic congestion become worse in cosmopolitan city, such as lower transportation efficiency, time loss, and air pollution [1, 2, 3]. One of the most significant reasons is that the traffic light management system doesn't satisfy the modern traffic flow need. In conventional traffic light control model, model with fixed timing and fixed cycle is used [4]. If the fluctuation of traffic volume is in the tolerance of design volume, the problem will not show. Once the peak of volume exceeds the design volume, the congestion becomes serious and can't relieve. As the volume increased, especially, the peak is far more than the design flow, most of the historic cities also use the fixed timing traffic control systems that can't meet the large or dynamic traffic requirements, and the congestion lead by the light is serious. Therefore, some improvement measures must carry out to relieve or solve this problem. Some scholars, who take the real time traffic volume into account, have done lots of research work targeting to reduce the delay and improve traffic volume in the intersection $[5,6]$.

The variable traffic light cycle and phase time based on the vehicle volume detection is more attracted and the new trend[7, 8], which fully considers dynamic volume and makes the light control more feasible for improving max traffic volume and reducing waiting time after the red light. W. Wen proposed a framework for a dynamic and automatic traffic light control expert system combined with a simulation model to analyze the traffic problem [7]. Some studies based on fuzzy logic are obtaining optimal traffic flow to reach minimal waiting time $[9,10]$. Wireless communication between cars and between car and road side units can be used to improve the traffic flow control as well as traffic safety $[11,12]$.

X. H. Yu proposed an adaptive control model for network of signalized intersections based on a discrete-time, stationary, Markov decision process. Simulation results using this model show significant improvement over traditional full-actuated control, especially for the case of high volume [13]. S. Kwatirayo, et al proposed a new adaptive traffic light control algorithm based on Vehicular Ad-Hoc Network, and test the algorithm in a specific intersection in the city of Moncton with real traffic data. The results show a substantial improvement of traffic throughput and average waiting time in comparison with fixed optimal cycle's time [14]. Lammer S, Liu Z used intelligent methods to implement the urban traffic light control in road network $[15,16]$. Space division are also the most 
used methods, which can effectively reduce delay and increase the volume of crossing. But it needs great investment to build infrastructure.

Fixed cycle and fixed phase time is usually used in most grade crossings, which works very well in the condition of small traffic volume with low variance. Once the traffic volume varies from small to very large, some traffic chaos would happen. In small volume, waste of the effective green time is serious, which comparatively leads to the delay increase of other phases. And in large volume situation, the queue waiting to pass the intersection is so long that the green time was not enough to let all vehicles to pass. The reflected wave of volume would lead to longer queue. However, the condition is not always satisfying the requirement, especially for space limitation intersection.

In modern city, the traffic volume varies very large in different directions at different time. The traffic light with fixed pass time can't meet the vehicles waiting in the queue to pass, which may lead to serious long queue, traffic interrupt or traffic gridlock. Mirchandani P \& Head L proposed the software architecture, solution algorithm and analysis of a real-time traffic-adaptive signal control system. The control system the real-time vehicle detection data as input and the simulation results show promising experimentation [17]. Research on the dynamic traffic light control system according to the real-time queue length is worth studying, which is more practical for modern signal intersection, and can improve the efficiency of intersection traffic and minimize the queue delay.

Based on presentation above, a method calculating the traffic light time based on queue length in a special phase is proposed. The vehicle detecting sensors were utilized to measure the vehicle average speed and the queue length. According to sensors data, a mode of dynamical adjustment of the phase time was setup. Furthermore, the pedestrian and bicycle are also taken into account.

\section{Data Collection System}

Two type sensors (inductive-loop detector and magnetic field sensor) are used for measuring the parameters of number of vehicle waiting in the queue, queue length and average speed of the vehicle passing the intersection.

\subsection{Vehicle Detection System}

The presence and movement of vehicles is detected by the magnetic sensor [18]. The Honeywell's magneto-resistive sensor HMC1022 is a low power consumption, low cost, high precision and sensitivity product, and suitable for detecting the earth magnetic anomaly caused by the ferrous material of vehicle chassis. If the vehicle passes on the sensor, the volume and presence of vehicle can be measured, as shown in Fig.1. The output of the magnetic sensor is not strong enough and sinusoidal function with low noise, so the signal is amplified before converting to rectangle wave. Fig. 2 shows the complete processing circuit.

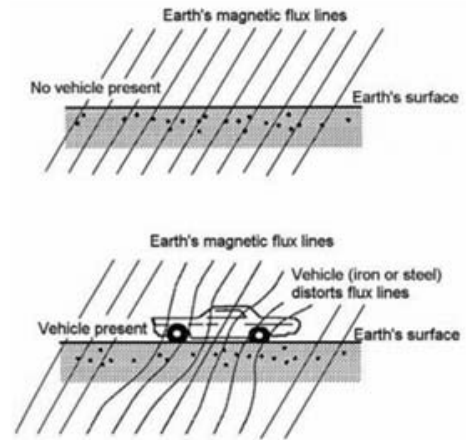

Fig.1 Principle of detection

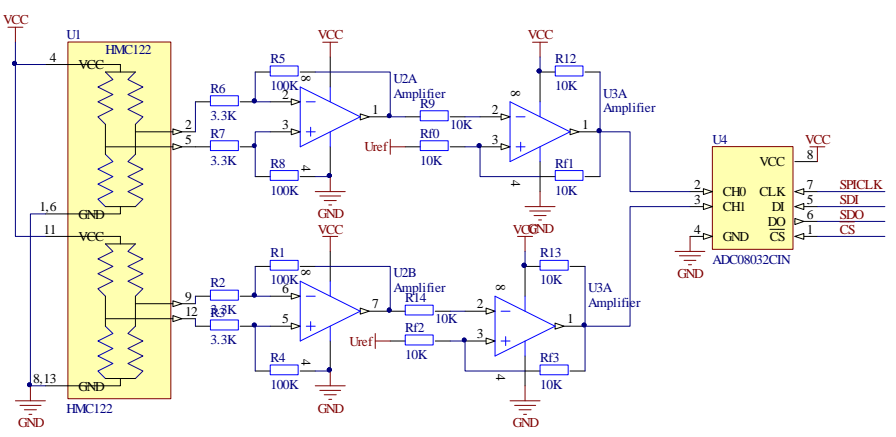

Fig.2 Vehicle detection circuit of HMC1022

\subsection{Vehicle Speed Measurement}

The method of vehicle speed measurement is testing the time $t$ the vehicle passes through a certain distance $L$. The velocity $v$ is $v=L / t$. As Fig. 3 shows, two inductive-loop sensors are placed at a known short distance, when the vehicle passes the first inductive-loop sensor, a pulse is produced to trigger the control system to start timing [19]. And when the vehicle passes the second sensor, another pulse is produced to stop timing. The time the vehicle enters the two sensors can be concluded, so the 
speed of vehicle can be obtained. The inductive-loop and the capacity on the control system form a LC resonance circuit. The resonance frequency of LC circuit without vehicle on the loop is approximate $20 \mathrm{kHz}$, which becomes $80 \sim 120 \mathrm{kHz}$ when the vehicle on the loop. Therefore, the presence or pass of the vehicle can be detected according to the resonance frequency. The signal from the LC circuit is sinusoidal wave, which should be converted to rectangle wave so as to process it with microcontroller. Transistors of Q1 and Q2 work in status of saturation distortions, so the positive peak of the sin wave is cut off; while the Q3 and Q4 work in status of cutoff distortion, so the negative peak of the sin wave is chopped. Then the sin wave becomes rectangle wave, which can directly input to pin of microcontroller timer, the whole processing course shows in Fig. 4.

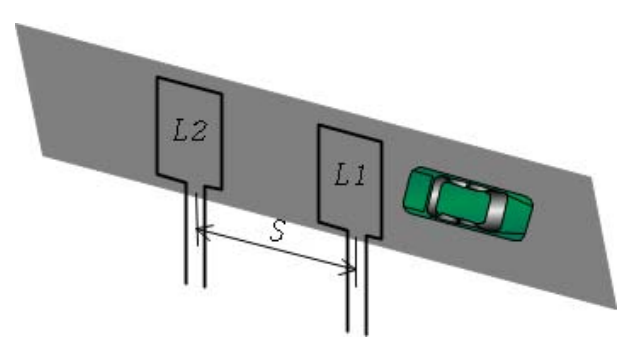

Fig.3 Speed measurement of vehicle

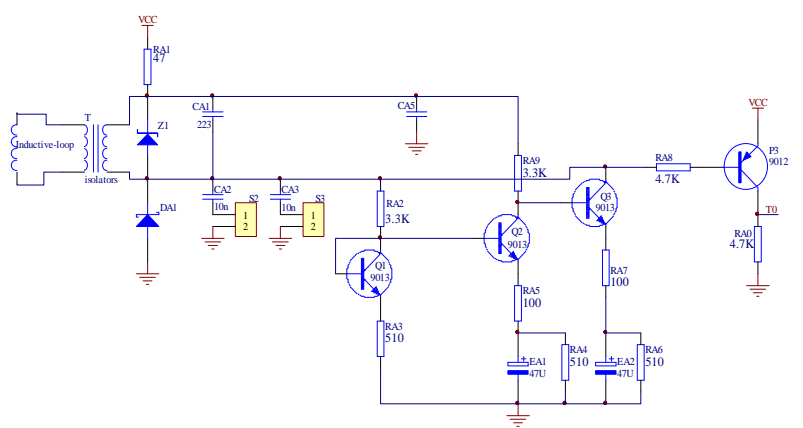

Fig.4 Vehicle detecting circuit

\section{Model of Vehicle Queue}

\subsection{Boundary Conditions of The Model}

As Fig.5 shows a normal intersection, all the phases are permitted, but there is nothing restriction for turning right. The sensors in the presence detection position detect the vehicle presence and the number of vehicle passed in current cycle, which can be used to evaluate the queue length left. If there are vehicle stopping on the sensors placed in the minimal distance, the queue length is longer than the minimal distance (The minimal distance is set for calculating the minimal safe time $t_{\min }$ for pedestrians to pass through the intersection, therefore, even if there is none vehicle stop on the presence detection position, the green light time can't be less than $t_{\min }$ ). In order to improve transportation efficiency and reduce the queue waiting time of other phases, a maximal green light time $t_{\max }$ is limited. Between $t_{\min }$ and $t_{\max }$, the green light time can be adjusted according to the real-time queue length.

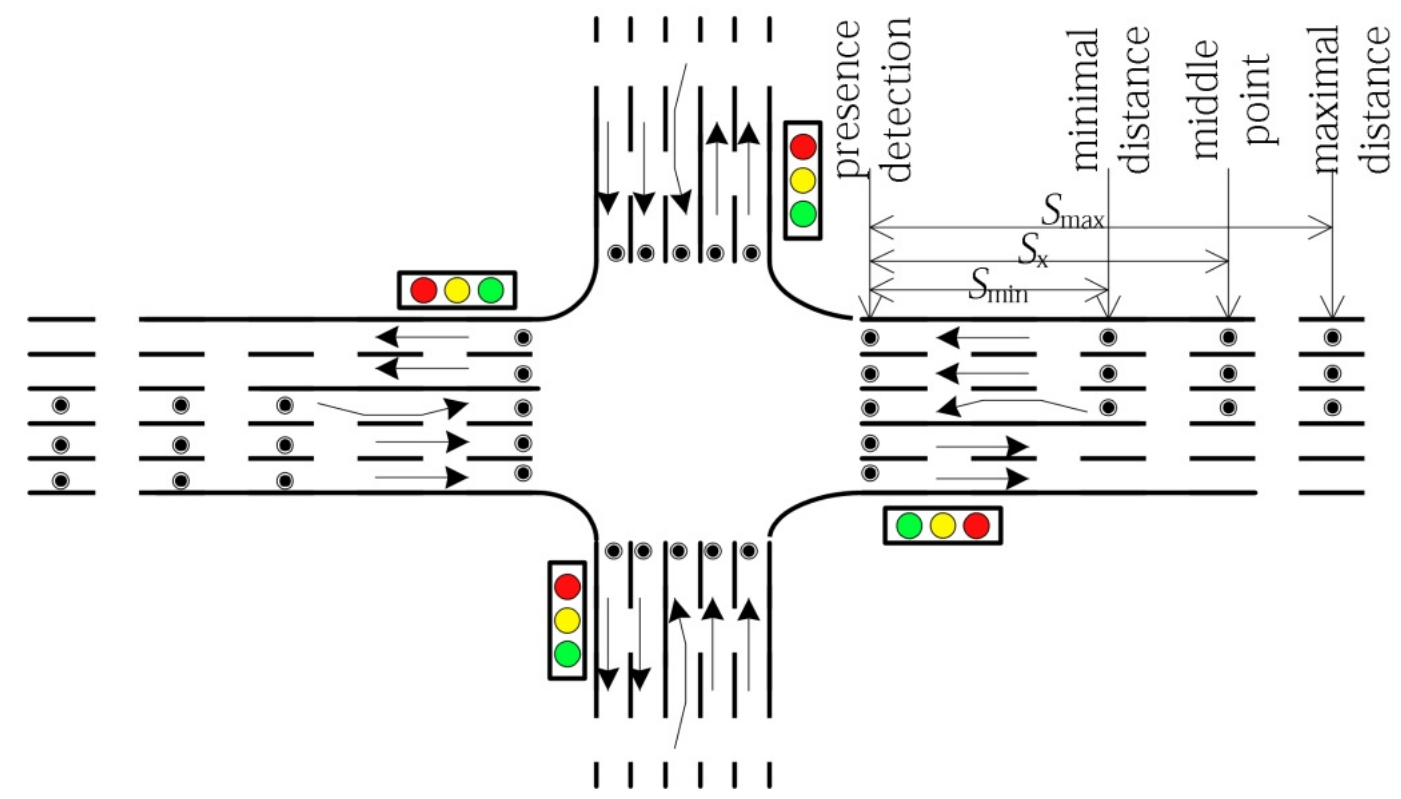

Fig.5 Intersection model with magnetic detectors 


\subsection{Light Time Adjustment Model}

The sensors placed between the minimal distance and the maximal distance are used to detect the real-time queue length and volumes, if the light time satisfies the boundary constrains, the light time can be adjustable. Fig. 5 and Fig. 6 show the calculating model.

The model discussed below is based on some hypothesis: (1) the vehicles passing the max length detector don't change its lane; (2) the traffic flow is considered as stationary flow without extraordinarily disturbance; (3) the intrusion among different phases is not thought about.

Assumed that: the average velocity of the queue is $v_{\text {avr }}$, the average vehicle length(including distance headway) is $l_{\text {avr }}$, the real-time queue length is $L_{\mathrm{rt}}$, the number of vehicle passing through the maximal distance is $N_{\text {new }}$ and the number of vehicle passed is $N_{\text {passed }}$. Therefore, the number of vehicle waiting to pass is

$$
N_{x}=\frac{L_{r t}}{l_{\text {avr }}}+N_{\text {in }}-N_{\text {passed }}
$$

It is assumed that the vehicles passing through the maximal distance during the passing cycle can reach the end of the queue, so the time needed to pass all the vehicle is

$$
t_{x}=\frac{N_{x} l_{\text {avr }}}{v_{\text {avr }}}=\frac{L_{r t}+N_{\text {in }} l_{\text {avr }}-N_{\text {passed }} l_{\text {avr }}}{v_{\text {avr }}}
$$

$L_{\mathrm{rt}}$ is determined by presence and advance detector point. Assumed that the rear of the queue is between $S_{i}$ and $S_{i+1}(0<\mathrm{i}<\mathrm{n})$, so the sensors in the $S_{i}$ detect the steady moving queue, but the sensors in the $S_{i+1}$ don't detect steady vehicle volume, therefore, the $L_{\mathrm{rt}}$ can be expressed as

$$
L_{r t}=\sum_{j=1}^{i} S_{j}+S_{a d j}, \quad i \geq j
$$

Where, $S_{a d j}$ is the evaluated value, between $0 \leq S_{a d j} \leq S_{i+1}$.

During the passing time, there may be a considerable amount of vehicle queued, $t_{x}$ will be greater than the time upboundary restrictions, but its maximal value is $t_{\max }$.

In reality, the vehicles passing through the maximal point may not reach the end of the queue and waiting for these vehicles to passing the intersection may waste the time of other phases, thus, if the number of vehicles is less than a given threshold, these new coming vehicles are not taken into account in the current cycle. Or some optimizing work can be done at this situation

In summary, the green light time $t_{g}$ can be expressed as

$$
t_{g}=\left\{\begin{array}{lr}
t_{\text {min }}, & t_{x} \leq t_{\text {min }} \\
t_{x}, & t_{\text {min }}<t_{x}<t_{\text {max }} \\
t_{\text {max }}, & t_{x} \geq t_{\text {max }}
\end{array}\right.
$$

However, equation 4 must satisfy the time constrains.

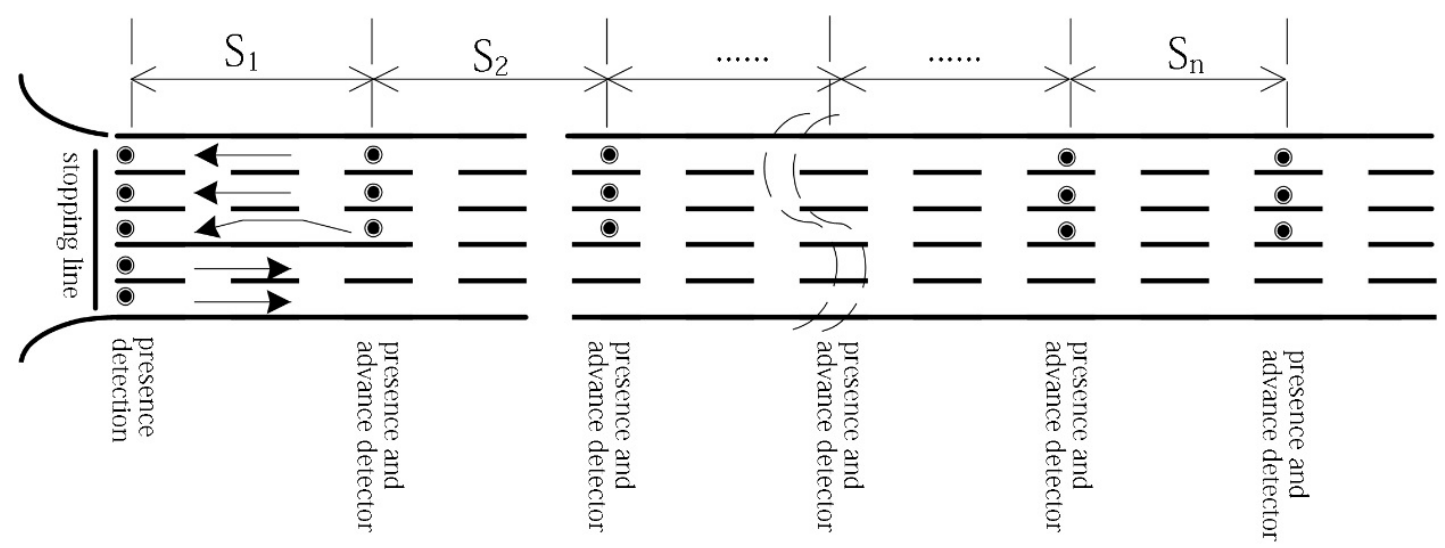

Fig.6 Model for deciding the real-time vehicle queue length

\section{Conclusion and Future Work}

In the paper a feasible timing model of traffic light based on the characteristics of real-time vehicle flow was proposed. Two type sensors was used to detect the key parameters of the queue such as queue length, number of vehicles waiting in the queue, and the average speed. The mathematic model 
setup was a common crossing model, and the pedestrian and bicycle rider were taken into account. Therefore, it was useful to analyze the traffic light system or simulate.

The method described above mainly focused on the algorithm of one phase, and was not taken the other phases into account, it is necessary to balance the volume among different phases when using the variable light time and optimize the traffic light system to maximize the effectiveness of whole intersection.

\section{References}

[1]. Dresner K, Stone P. Multiagent traffic management: An improved intersection control mechanism[C]. Proceedings of the fourth international joint conference on Autonomous agents and multiagent systems. ACM, 2005: 471-477.

[2]. Wang F Y. Integrated intelligent control and management for urban traffic systems[C]. Intelligent Transportation Systems, 2003. Proceedings. IEEE, 2003, 2: 1313-1317.

[3]. US Department of transportation (2007). Congestion Mitigation. http://www.fhwa.dot.gov/ congestionmitigation/congestionmitigation.htm.

[4]. Cihan Karakuzu, Osman Demirci. Fuzzy logic based smart traffic light simulator design and hardware implementation [J]. Applied Soft Computing, 2010, 10: 66-73.

[5]. Fouladvand M E, Sadjadi Z, Shaebani M R. Optimized traffic flow at a single intersection: traffic responsive signalization [J]. Journal of Physics A: Mathematical and General, 2004, 37(3): 561.

[6]. Mirchandani P, Head L. A real-time traffic signal control system: architecture, algorithms, and analysis [J]. Transportation Research Part C: Emerging Technologies, 2001, 9(6): 415-432.

[7]. W. Wen. A dynamic and automatic traffic light control expert system for solving the road congestion problem [J]. Expert Systems with Applications, 2008, 34: 2370-2381.

[8]. Chen, Y. L., \& Yang, H. H. Minimization of travel time and weighted number of stops in a traffic-light network [J]. European Journal of Operational Research, 2003, 144:565-580.

[9]. C.-H. Chou, J.-C. Teng. A fuzzy logic controller for traffic junction signals [J]. Information Sciences, 2002, 143:73-97.

[10]. J. Niittyma"ki, E. Turunen. Traffic signal control on similarity logic reasoning [J]. Fuzzy Sets and Systems, 2003, 133:109-131.

[11]. Nitin Maslekar, Joseph Mouzna, Mounir Boussedjra, Houda Labiod. CATS: An adaptive traffic signal system based on car-to-car communication [J]. Journal of Network and Computer Applications, 2013, 36:1308-1315.

[12]. Gupte S, Younis M. Vehicular networking for intelligent and autonomous traffic management[C]. Communications (ICC), 2012 IEEE International Conference on. IEEE, 2012: 5306-5310.

[13]. Yu X H, Recker W W. Stochastic adaptive control model for traffic signal systems [J]. Transportation Research Part C: Emerging Technologies, 2006, 14(4): 263-282.

[14]. Kwatirayo S, Almhana J, Liu Z. Adaptive Traffic Light Control using VANET: A case study[C]. Wireless Communications and Mobile Computing Conference (IWCMC), 2013 9th International. IEEE, 2013: 752-757.

[15]. Lammer S, Helbing D. Self-control of traffic lights and vehicle flow in urban road networks [J]. International Journal of Statistical Mechanics: Theory and Experiment, 2008:1-36. 
[16]. Liu Z. A survey of intelligent methods in urban traffic signal control [J]. International Journal of Computer Science and Network Security, 2007, 7:105-12.

[17]. Mirchandani P, Head L. A real-time traffic signal control system: architecture, algorithms, and analysis [J]. Transportation Research Part C: Emerging Technologies, 2001, 9(6): 415-432.

[18]. Cheung S Y, Coleri S, Dundar B, et al. Traffic measurement and vehicle classification with single magnetic sensor [J]. Transportation research record: journal of the transportation research board, 2005, 1917(1): 173-181.

[19]. Robinson S, Polak J W. Modeling urban link travel time with inductive loop detector data by using the k-NN method [J]. Transportation Research Record: Journal of the Transportation Research Board, 2005, 1935(1): 47-56. 